\title{
Nutritional intake and dietary patterns in pregnancy: a longitudinal study of women with lifetime eating disorders
}

\author{
Nadia Micali ${ }^{1 *}$, Kate Northstone ${ }^{2}$, Pauline Emmett ${ }^{2}$, Ulrike Naumann ${ }^{3}$ and Janet L. Treasure ${ }^{4}$ \\ ${ }^{1}$ Behavioural and Brain Sciences Unit, Institute of Child Health, University College London, 4th Floor, \\ 30 Guilford Street, London WC1N 1EH, UK \\ ${ }^{2}$ School of Social and Community Medicine, University of Bristol, Oakfield House, Oakfield Grove, \\ Bristol BS8 2BN, UK \\ ${ }^{3}$ Department of Biostatistics, Institute of Psychiatry, King's College London, De Crespigny Park, \\ London SE5 $8 A F$, UK \\ ${ }^{4}$ Eating Disorders Research Unit, Department of Psychological Medicine, Institute of Psychiatry, \\ King's College London, London, UK
}

(Submitted 24 August 2011 - Final revision received 11 January 2012 - Accepted 16 January 2012 - First published online 12 July 2012)

\section{Abstract}

There is limited knowledge about dietary patterns and nutrient/food intake during pregnancy in women with lifetime eating disorders (ED). The objective of the present study was to determine patterns of food and nutrient intake in women with lifetime ED as part of an existing longitudinal population-based cohort: the Avon Longitudinal Study of Parents and Children. Women with singleton pregnancies and no lifetime psychiatric disorders other than ED ( $n$ 9723) were compared with women who reported lifetime (ever) ED: (anorexia nervosa (AN, $n$ 151), bulimia nervosa (BN, $n$ 186) or both (AN $+\mathrm{BN}, n$ 77)). Women reported usual food consumption using a FFQ at 32 weeks of gestation. Nutrient intakes, frequency of consumption of food groups and overall dietary patterns were examined. Women with lifetime ED were compared with control women using linear regression and logistic regression (as appropriate) after adjustment for relevant covariates, and for multiple comparisons. Women with lifetime ED scored higher on the 'vegetarian' dietary pattern; they had a lower intake of meat, which was compensated by a higher consumption of soya products and pulses compared with the controls. Lifetime AN increased the risk for a high ( $\geq 2500 \mathrm{~g} /$ week) caffeine consumption in pregnancy. No deficiencies in mineral and vitamin intake were evident across the groups, although small differences were observed in macronutrient intakes. In conclusion, despite some differences in food group consumption, women with lifetime ED had similar patterns of nutrient intake to healthy controls. Important differences in relation to meat eating and vegetarianism were highlighted, as well as high caffeine consumption. These differences might have an important impact on fetal development

\section{Key words: Nutrition: Pregnancy: Eating disorders}

The eating disorders (ED) anorexia nervosa (AN) and bulimia nervosa $(\mathrm{BN})$, and eating disorder not otherwise specified affect about $5-7 \%$ of women of childbearing age. There is an increasing literature from large cohorts and data registers showing clear effects of active and past ED on obstetric outcomes $^{(1-4)}$. These studies all point to a lower birth weight, especially in the offspring of women with lifetime AN. Although previous studies have shown that ED symptoms tend to improve during pregnancy ${ }^{(1,2)}$, only one study to date has investigated exact dietary intakes in pregnancy in women with active/past (before pregnancy) $\mathrm{ED}^{(5)}$. This large study on a population sample of Norwegian pregnant women showed differences in early pregnancy in food group intakes in women with active $\mathrm{BN}$ compared with controls and higher nutritional intakes in women with active binge eating disorder. Although this well-conducted study investigated women with active or last 6 months' history of binge eating disorder and BN, no subjects with active/past AN were available for study.

Clinical experience highlights an uncertainty among women with active/past ED in relation to nutritional requirements in pregnancy. We previously highlighted an important role for pre-pregnancy BMI in mediating the effect of maternal AN on birth weight ${ }^{(1)}$. Dietary consumption in pregnancy might also be an important factor contributing to adverse

Abbreviations: ALSPAC, Avon Longitudinal Study of Parents and Children; AN, anorexia nervosa; AN + BN, both anorexia and bulimia nervosa; BN, bulimia nervosa; ED, eating disorder. 
fetal outcomes ${ }^{(6)}$. Therefore, clarifying differences in nutrient and food group consumption and overall dietary patterns in pregnancy in women with lifetime ED could help clarify the risk pathways.

The present study aims to build on and complement the evidence provided by Siega-Riz et al. ${ }^{(5)}$ by investigating the frequency of consumption of various food groups and quality of intake (macronutrient intakes) in a large general population cohort based in the UK: the Avon Longitudinal Study of Parents and Children (ALSPAC). Due to the high correlation and biological interaction between food and nutrient intakes, we were also interested in studying dietary patterns of pregnant women with lifetime ED obtained using principal components analysis to identify underlying dietary patterns in the data.

We hypothesised that women with lifetime ED would have a lower energy, lower sugar and lower fat intake compared with unexposed women.

\section{Subjects and methods}

\section{Participants and procedures}

The ALSPAC is a longitudinal, prospective study designed to examine the effects of environment, genetics and other factors on health and development ${ }^{(7)}$. All pregnant women living in the geographical area of Avon, UK, who were expected to deliver their baby between 1 April 1991 and 31 December 1992, were recruited; 14472 women were enrolled. Primary data collection was via self-completion questionnaires.

At 12 weeks of gestation, women were asked whether they had ever had any psychiatric problems, including depression, schizophrenia, alcohol abuse, AN, BN or any other disorder. Women were excluded from the present study if they had not completed this questionnaire ( $n$ 2019), or if they reported in this questionnaire any lifetime history of psychiatric problems (other than ED) only ( $n$ 1166), as detailed data on psychopathology in this group were lacking. Women were excluded if they had non-singleton pregnancies ( $n$ 199) and if they had a miscarriage for the current pregnancy ( $n$ 36).

The final sample eligible for the study was 11052 women. Information on ethnicity and maternal education was missing for 691 women (6.2\%). Complete baseline and dietary data were available for 10137 (91.7\%) women.

Women were divided into four groups according to their answer to the 12 weeks questionnaire on having ever had $\mathrm{AN}, \mathrm{BN}$ or both ${ }^{(1)}$ : women with a self-reported lifetime AN ( $n$ 151); women with a self-reported lifetime BN ( $n$ 186); women who reported both lifetime $\mathrm{AN}$ and $\mathrm{BN}(n$ 77); a group of unexposed women representative of the general population ( $n$ 9723).

\section{Measures}

Sociodemographic and weight and height data were obtained by a self-completion questionnaire at 12, 18 and 32 weeks of gestation; BMI was calculated as pre-pregnant weight (kg)/ height $\left(\mathrm{m}^{2}\right)$. Dietary information was collected via the FFQ, completed by women at 32 weeks of gestation.

\section{Food group consumption}

The FFQ contained a set of questions enquiring about the frequency of consumption of a wide variety of foods and drinks. The women were given the following options to indicate how often they were currently consuming a variety of food types: (1) never or rarely; (2) once in 2 weeks; (3) 1-3 times/ week; (4) 4-7 times/week; (5) more than once daily. The women were also asked to record how many cups of tea or coffee, the number of glasses of cola and the number of slices of bread they usually consumed daily. Dietary supplements were not included in the FFQ. The frequency of consumption data were numerically transformed into times consumed per week, in order to apply quantitative meaning to the frequency categories, as follows: (1) 0 , (2) 0.5 , (3) 2 , (4) 5.5 and (5) 10 times/week.

In order to summarise dietary data, we grouped foods, where it could be done in a meaningful way. For example, frequencies of eating red meat, poultry, sausages/burgers and pies/pasties were summed into a variable called 'all meat'. 'Fish' included white fish, oily fish and shellfish. 'Potatoes' included chips, roast potatoes and boiled or baked potatoes, and 'vegetables' included peas, maize or similar, cabbage or similar, green vegetables, carrots, root vegetables and salad. Fruit and fruit juice were combined. 'Sweets/chocolate/ cakes/biscuits' included puddings, cakes or buns, chocolates, sweets, biscuits and chocolate bars. Soya and meat substitutes were also summed together. Women were also asked to indicate whether they were vegetarian or ate meat.

\section{Dietary patterns}

Dietary patterns were obtained from the FFQ (detailed above) using principal components analysis. This has been described in detail elsewhere ${ }^{(8)}$. Briefly, the number of components best representing the data was primarily chosen on the basis of a scree plot and the interpretability of the components. Women were excluded from the principal components analysis if they had more than ten dietary items missing. If ten or fewer items were missing, we made the assumption that the women did not consume those items and they were given a value of 0 . A component score was created for each woman for each of the components identified, calculated by multiplying the factor loadings by the corresponding standardised value for each food and summing across the food items. Each score had a mean of 0 and a higher score indicated closer adherence to that dietary pattern. The following five components were obtained: 'health conscious' (high loadings for salad, fruit, rice, pasta, oat and bran-based breakfast cereals, fish, pulses, fruit juices and non-white bread); 'traditional' (high consumption of all types of vegetables and red meat and poultry); 'processed' (high intakes of high-fat processed foods, such as meat pies, sausages and burgers, fried foods, pizza, chips and baked beans); 'confectionery' (high intakes of foods with high sugar content such as chocolate, sweets, biscuits, cakes and other puddings); 'vegetarian' (high loadings for meat substitutes, pulses, nuts and herbal teas, and high negative loadings for red meat and poultry). 


\section{Nutrients}

Daily nutrient intakes were estimated from the FFQ using the 5th edition of McCance and Widdowson's 'The Composition of Food' and supplements based on standard portion sizes; detailed information on the methodology has been published elsewhere ${ }^{(9)}$. Previous analysis of these data ${ }^{(9)}$ showed this questionnaire to produce mean nutrient intakes similar to those obtained for women in the British National Diet and Nutritional survey for adults ${ }^{(10)}$. Data for analysis of nutrient intakes were missing for forty-eight women (0.5\%) and available for 10073 women (91.1\%). Missingness was independent of exposure status.

\section{Statistical analyses}

Preliminary analyses determined distribution patterns of all variables; variables that were found to be skewed underwent logarithmic transformation before analyses. Group comparisons used parametric (one-way ANOVA) tests as appropriate, after testing for normality. Multinomial and binary logistic regression models examined predictors of categorical and binary outcomes, respectively.

Potential covariates previously found to be associated with relevant outcomes in this sample ${ }^{(8)}$ and likely to influence outcomes were first tested in bivariate models and then included in multivariate models if associated with the outcomes. The final model accounted for main effects of each covariate. All analyses of nutrient intakes were adjusted for total energy intake. All analyses were performed using Stata 10 for Windows $^{(11)}$. All statistical tests presented are two-tailed. Statistical significance was defined as a $P$ value of less than $0 \cdot 05$.

\section{Multiple testing}

In order to take into account the effect of multiple testing on potential chance significant findings, two steps were taken. First, women with lifetime ED were compared with controls on selected outcomes; only if a difference was found were comparisons extended to ED subgroups ( $\mathrm{AN}, \mathrm{BN}$ and $\mathrm{AN}+\mathrm{BN}$ ). At this second stage of analyses, the Bonferroni-Holm ${ }^{(12)}$ procedure was used to adjust for multiple testing. This procedure is more powerful and less conservative than a Bonferroni correction and allows adjusting $P$ values obtained during multiple testing.

We did not apply a method to adjust for multiple testing across outcomes, as this would have reduced power to an unacceptable level, and as this is an exploratory study. Instead, we have provided significance for different levels of type I error ${ }^{(13)}$.

\section{Ethics}

The present study was approved by the Institute of Psychiatry Ethics Committee (reference no. 110/02), the ALSPAC Law and Ethics Committee and the Local Research Ethic Committees. All women gave written informed consent for participation in the study.

\section{Results}

\section{Sociodemographic data}

Age at delivery, parity and ethnic distribution were comparable across the groups. Women with lifetime $\mathrm{AN}$ and $\mathrm{AN}+\mathrm{BN}$ were more likely to have obtained A-levels compared with unexposed women (OR 1.6, 95\% CI 1·2, 2.2; OR 2.2, $95 \%$ CI $1 \cdot 4$, $3 \cdot 4$, respectively; see Table 1 ).

\section{Food group consumption and dietary patterns}

Women with ED were $2 \cdot 8$ times more likely to describe themselves as vegetarian (OR $2 \cdot 8,95 \%$ CI $2 \cdot 1,3 \cdot 8$ ) compared with unexposed women; all subgroups differed from the unexposed (AN: OR 2.8, 95\% CI 1.7, 4.4; BN: OR 2.3, $95 \%$ CI 1.4, 3.7; $\mathrm{AN}+\mathrm{BN}$ : OR $4 \cdot 3,95 \%$ CI $2 \cdot 3,7 \cdot 7)$.

Women with ED consumed less meat than unexposed women $(b$ coefficient $-1 \cdot 6$, 95\% CI $-2 \cdot 1,-1 \cdot 1 ; P<0.001$ (respectively AN: $-1 \cdot 8,95 \% \mathrm{CI}-2 \cdot 6,-1 \cdot 0$; $\mathrm{BN}:-1 \cdot 4,95 \%$ $\mathrm{CI}-2 \cdot 1,-0 \cdot 7 ; \mathrm{AN}+\mathrm{BN}:-1 \cdot 8,95 \% \mathrm{CI}-2 \cdot 9,-0 \cdot 6)$, and fewer potatoes ( $b$ coefficient $-0.6,95 \%$ CI $-0.9,-0.2$ ). However, they ate more pulses ( $b$ coefficient $0 \cdot 2,95 \% \mathrm{CI}$ $0 \cdot 1,0 \cdot 3)$. Women with lifetime ED were more likely to consume soya and soya products ( $b$ coefficient $0 \cdot 3,95 \%$ CI $0 \cdot 2$, $0 \cdot 5$; see Table 2).

Table 1. Sociodemographic data: comparisons between index groups and unexposed women from ANOVA and logistic regression (Percentages, odds ratios and $95 \%$ confidence intervals)

\begin{tabular}{|c|c|c|c|c|c|c|c|c|c|c|c|c|}
\hline & \multicolumn{3}{|c|}{ AN $(n 151)$} & \multicolumn{3}{|c|}{$\mathrm{BN}(n 186)$} & \multicolumn{3}{|c|}{$\mathrm{AN}+\mathrm{BN}(n 77)$} & \multicolumn{3}{|c|}{ General population ( $n$ 9723) } \\
\hline & $\%$ & OR & $95 \% \mathrm{Cl}$ & $\%$ & OR & $95 \% \mathrm{Cl}$ & $\%$ & OR & $95 \% \mathrm{Cl}$ & $\%$ & OR & $95 \% \mathrm{Cl}$ \\
\hline \multicolumn{13}{|l|}{ Age at delivery (years) $\dagger$} \\
\hline Mean & $29 \cdot 1$ & & & $28 \cdot 2$ & & & $29 \cdot 4$ & & & $28 \cdot 4$ & & \\
\hline $\begin{array}{l}\text { SD } \\
\text { Parity (multiparous) }\end{array}$ & $5 \cdot 3$ & & & $\begin{array}{l}4 \cdot 6 \\
50 \cdot 0\end{array}$ & & & $\begin{array}{l}4.4 \\
53.9\end{array}$ & & & $\begin{array}{l}4.7 \\
54.9\end{array}$ & & \\
\hline $\begin{array}{l}\text { Parity (multiparous) } \ddagger \\
\text { Ethnicity (white) } \ddagger\end{array}$ & $\begin{array}{l}50 \cdot 3 \\
96 \cdot 2\end{array}$ & $\begin{array}{l}0.8 \\
0.6\end{array}$ & $\begin{array}{l}0 \cdot 6,1 \cdot 2 \\
0.3,1 \cdot 4\end{array}$ & $\begin{array}{l}50 \cdot 0 \\
97 \cdot 4\end{array}$ & $\begin{array}{l}0.8 \\
0.9\end{array}$ & $\begin{array}{l}0 \cdot 6,1 \cdot 1 \\
0 \cdot 4,2 \cdot 2\end{array}$ & $\begin{array}{l}53.9 \\
98.8\end{array}$ & $\begin{array}{l}0.9 \\
1.9\end{array}$ & $\begin{array}{l}0 \cdot 6,1 \cdot 5 \\
0.3,13 \cdot 8\end{array}$ & $\begin{array}{l}54 \cdot 9 \\
97 \cdot 6\end{array}$ & $\begin{array}{l}\text { Reference } \\
\text { Reference }\end{array}$ & \\
\hline $\begin{array}{l}\text { Education (A-levels or higher } \\
\text { v. up to O-levels) } \ddagger\end{array}$ & $49 \cdot 0^{*}$ & 1.6 & $1 \cdot 2,2 \cdot 2$ & $41 \cdot 1$ & $1 \cdot 2$ & $0.9,1.6$ & $56 \cdot 3^{\star \star \star}$ & $2 \cdot 2$ & $1 \cdot 4,3 \cdot 4$ & $36 \cdot 9$ & Reference & \\
\hline
\end{tabular}

AN, anorexia nervosa; $\mathrm{BN}$, bulimia nervosa; $\mathrm{AN}+\mathrm{BN}$, both anorexia nervosa and bulimia nervosa.

Values are shown for comparisons between each index group and unexposed women: ${ }^{\star} P<0.05,{ }^{\star \star \star} P<0.001$ (ANOVA, binary logistic regression).

$\dagger F=2.4(\mathrm{df}=3)$; ANOVA.

$\ddagger$ Logistic regression. 
Women with ED consumed greater amounts of bread per d: on average, $1 \cdot 1$ slices/d more than women in the general population ( $b$ coefficient $1 \cdot 1,95 \%$ CI $0 \cdot 2,1 \cdot 9$ ); this difference was evident for women with $\mathrm{BN}$ ( $b$ coefficient 1.9 , 95\% CI $0 \cdot 6,3 \cdot 2$ ).

Women with ED scored higher on the 'vegetarian pattern' compared with unexposed women ( $b$ coefficient $0 \cdot 3,95 \%$ CI $0 \cdot 2,0 \cdot 4 ;$ see Table 2). This association was consistent across the three ED subgroups. Women with lifetime $\mathrm{AN}+\mathrm{BN}$ had higher scores on the 'traditional' dietary pattern (b coefficient $0 \cdot 3,95 \% \mathrm{CI} 0 \cdot 1,0 \cdot 5$ ) and the 'health conscious' pattern ( $b$ coefficient $0 \cdot 2,95 \%$ CI $0 \cdot 01,0 \cdot 4$ ) compared with the unexposed. Women with lifetime AN scored higher on the 'traditional' dietary pattern ( $b$ coefficient $0 \cdot 2,95 \%$ CI $0.02,0.3$ ) compared with unexposed women (see Table 2; Table S1, available online).

\section{Use of fats, milk and caffeine}

Women with lifetime ED were less likely to use butter compared with unexposed women (OR 0.7, $95 \%$ CI 0.5, 0.8); however, they used margarine, low-fat spread and vegetable oil similarly to unexposed women. A lower use of full-fat milk (OR 0.7, 95\% CI 0.6, 0.9) and a higher use of skimmed milk (OR 1.4, 95\% CI 1.1, 1.8) and soya milk (OR 3.1, 95\% CI $1 \cdot 8,5 \cdot 2)$ compared with unexposed women were also evident.

A high weekly caffeine intake $(>2500 \mathrm{mg}$ ) was more common in women with lifetime ED than in the controls (OR 1.9, 95\% CI 1.3, 2.8); women with both lifetime AN and lifetime $\mathrm{AN}+\mathrm{BN}$ differed from unexposed women (OR $2 \cdot 6$, $95 \%$ CI $1 \cdot 4,4 \cdot 8$ and OR $2 \cdot 7,95 \%$ CI $1 \cdot 1,7 \cdot 0$ ) (see Table 3 ).

\section{Nutrient intake}

In relation to energy, carbohydrate, fat and protein consumption, no differences were highlighted between women with ED and unexposed women (see Table 4).

Across the ED subgroups, only minor differences in macronutrient consumption were observed (see Table S2, available online): a lower sugar and non-milk extrinsic sugar, and a higher polyunsaturated fat intake in women with lifetime BN. A lower intake of saturated fat was observed in women with lifetime $\mathrm{AN}$ and $\mathrm{AN}+\mathrm{BN}$. A higher fibre (NSP) intake was also observed in women with lifetime $\mathrm{AN}, \mathrm{BN}$ and $\mathrm{AN}+\mathrm{BN}$.

Mineral and vitamin intakes were not compromised in women with lifetime ED. Across the subgroups, all index groups had higher intakes of $\mathrm{Mg}$ and $\mathrm{Se}$; women with $\mathrm{AN}$ and $\mathrm{AN}+\mathrm{BN}$ of $\mathrm{Fe}$ and $\mathrm{K}$; and women with $\mathrm{AN}+\mathrm{BN}$ of $\mathrm{P}$, $\mathrm{Ca}$ and $\mathrm{Zn}$ compared with unexposed women.

Vitamin intake was higher for folate ( $b$ coefficient $10 \cdot 9,95 \%$ CI $2 \cdot 7,19 \cdot 1)$ in women with AN; lower in log-transformed retinol ( $b$ coefficient $-0 \cdot 1,95 \% \mathrm{CI}-0 \cdot 2,-0 \cdot 02)$ and higher in thiamin ( $b$ coefficient $0 \cdot 1,95 \%$ CI $0 \cdot 05,0 \cdot 2)$, vitamin C ( $b$ coefficient $14 \cdot 4,95 \%$ CI $7 \cdot 3,21 \cdot 4$ ) and folate ( $b$ coefficient $24 \cdot 2,95 \%$ CI $12 \cdot 8,35 \cdot 6)$ in women with lifetime $\mathrm{AN}+\mathrm{BN}$ compared with unexposed women (see Table S2, available online). Women with lifetime $\mathrm{BN}$ had a slightly higher vitamin $\mathrm{E}$ intake compared with the unexposed ( $b$ coefficient $0 \cdot 1,95 \%$ CI $0 \cdot 02,0 \cdot 15)$.

\section{Discussion}

This is the first study to investigate various aspects of dietary intake in the third trimester of pregnancy in women with lifetime ED using data from a large longitudinal general population study.

The present results show that, in general, dietary intake in pregnancy in women with lifetime ED was comparable to women from the general population without other psychiatric disorders. Some differences were highlighted in relation to food group consumption, in that women with lifetime ED consumed less meat and fewer potatoes in favour of soya products and pulses.

In relation to dietary patterns, there was a trend towards women with lifetime ED scoring higher on the 'health conscious' and 'traditional' dietary pattern, than unexposed women. In addition, women with ED scored higher on the 'vegetarian' dietary pattern and were three times more likely to describe themselves as vegetarians. This was reflected in more common use of soya milk in women with ED than in the unexposed. Siega-Riz et al. ${ }^{(5)}$ showed a lower intake of high-fat meats in the second trimester of pregnancy among women with BN before and during pregnancy in a large general population study.

Although there is evidence that a vegetarian diet is adequate in pregnancy and might actually result in positive infant health outcomes $^{(14)}$, maternal soya intake in pregnancy has been shown to have some effects on sexual maturation in animal studies $^{(15)}$, in particular earlier pubertal maturation in female offspring.

Women with lifetime ED were also less likely to use butter and drink full-fat milk (in favour of skimmed and soya milk). This is consistent with the 'vegetarian' dietary pattern highlighted.

Interestingly, women with lifetime ED were almost twice as likely to consume $\geq 2500 \mathrm{mg}$ caffeine/week (this is equivalent to about twenty-five cups of brewed coffee/week) compared with healthy women and higher than the recommendations of the Department of Health for pregnant women. This was particularly true for women with lifetime $\mathrm{AN}$ and $\mathrm{AN}+\mathrm{BN}$. The Food Standards Agency recommends that pregnant women should drink less than $200 \mathrm{mg}$ caffeine $/ \mathrm{d}^{(16)}$. Siega-Riz et al. ${ }^{(5)}$ showed a slight increase in mean coffee consumption in women with binge eating disorder during pregnancy in their study.

Previous studies on clinical and non-clinical samples have highlighted increased consumption of caffeine in women with ED and have suggested that this might be driven by a wish to suppress appetite and/or for its stimulating properties $^{(17,18)}$. Caffeine crosses the placenta and there is increasing evidence that caffeine intake in pregnancy affects the fetus. A detrimental effect of caffeine has been shown on fetal weight and length ${ }^{(19,20)}$

Few differences were evident in relation to macronutrient intake in women with lifetime ED. Across maternal ED, 
Table 2. Food group consumption (times/week) and dietary patterns from the FFQ: adjustedł ANOVA§ (B coefficients and $95 \%$ confidence intervals) and logistic regression (odds ratios and $95 \%$ confidence intervals) by group

\begin{tabular}{|c|c|c|c|c|c|c|c|c|c|}
\hline & \multicolumn{2}{|c|}{ ED $(n 414)$} & \multicolumn{2}{|c|}{ AN $(n 151)$} & \multicolumn{2}{|c|}{$\mathrm{BN}(n 186)$} & \multicolumn{2}{|c|}{$\mathrm{AN}+\mathrm{BN}(n 77)$} & \multirow[b]{2}{*}{ Unexposed ( $n$ 9723) } \\
\hline & $B$ & $95 \% \mathrm{Cl}$ & $B$ & $95 \% \mathrm{Cl}$ & $B$ & $95 \% \mathrm{Cl}$ & $B$ & $95 \% \mathrm{Cl}$ & \\
\hline Does not eat meat/vegetarian & & & & & & & & & Reference \\
\hline OR & \multirow{2}{*}{\multicolumn{2}{|c|}{$\begin{array}{c}2 \cdot 8^{\star * \star} \\
2 \cdot 1,3 \cdot 8\end{array}$}} & \multirow{2}{*}{\multicolumn{2}{|c|}{$\begin{array}{c}2 \cdot 8^{\star \star \star} \\
1 \cdot 7,4 \cdot 4\end{array}$}} & \multirow{2}{*}{\multicolumn{2}{|c|}{$\begin{array}{c}2.3^{\star *} \\
1.4 .3 .7\end{array}$}} & \multirow{2}{*}{\multicolumn{2}{|c|}{$\begin{array}{l}4 \cdot 3^{\star \star \star} \\
2.37 .7\end{array}$}} & \\
\hline $95 \% \mathrm{Cl}$ & & & & & & & & & \\
\hline All meat & $-1 \cdot 6^{\star \star *}$ & $-2 \cdot 1,1 \cdot 1$ & $-1 \cdot 8^{\star \star \star}$ & $-2 \cdot 6,-1 \cdot 0$ & $-1 \cdot 4^{\star \star \star}$ & $-2 \cdot 1,-0.7$ & $-1 \cdot 8^{\star \star \star}$ & $-2.9,-0.6$ & Reference \\
\hline Fish & $0 \cdot 3$ & $-0.1,0.7$ & \multicolumn{2}{|c|}{-} & \multicolumn{2}{|c|}{-} & \multicolumn{2}{|c|}{-} & Reference \\
\hline Potatoes & $-0.6^{\star \star}$ & $-0.9,-0.2$ & -0.4 & $-0.9,0.2$ & $-0.9^{\star \star \star}$ & $-1.4,-0.3$ & -0.4 & $-1 \cdot 1,0.4$ & Reference \\
\hline Vegetables & 0.5 & $-0.4,1.4$ & \multirow{2}{*}{\multicolumn{2}{|c|}{-}} & \multicolumn{2}{|c|}{-} & \multicolumn{2}{|c|}{-} & Reference \\
\hline Fruit and fruit juice & -0.1 & $-0.6,0.4$ & & & \multirow{2}{*}{\multicolumn{2}{|c|}{$\begin{array}{l}- \\
-\end{array}$}} & \multicolumn{2}{|c|}{-} & Reference \\
\hline All cereals (oat, bran, other) & -0.3 & $-1 \cdot 0,0 \cdot 3$ & \multicolumn{2}{|c|}{$\begin{array}{l}- \\
-\end{array}$} & & & \multicolumn{2}{|c|}{-} & Reference \\
\hline Sweets/chocolate/cakes/biscuits & $-1.3 \dagger$ & $-2 \cdot 2,-0.3$ & -1.4 & $-3 \cdot 0,0.2$ & -1.0 & $-2 \cdot 5,0.3$ & -1.5 & $-3 \cdot 7,0.8$ & Reference \\
\hline Pulses (log transformed) & $0 \cdot 2^{\star \star *}$ & $0.1,0.3$ & $0 \cdot 1^{\star *}$ & $0.1,0.3$ & $0 \cdot 1^{\star *}$ & $0.1,0.2$ & $0.4^{\star \star \star}$ & $0.2,0.6$ & Reference \\
\hline Soya or meat substitutes & $0.3^{\star \star \star}$ & $0.2,0.5$ & $0.3^{\star \star \star}$ & $0.1,0.5$ & $0.3^{\star \star}$ & $0.1,0.4$ & $0.5^{\star \star \star}$ & $0.2,0.8$ & Reference \\
\hline Bread (slices/d) & $1 \cdot 1 \dagger$ & $0.2,1.9$ & 0.3 & $-1 \cdot 2,1 \cdot 7$ & $1.9^{\star *}$ & $0.6,3.2$ & 0.4 & $-1 \cdot 6,2 \cdot 4$ & Reference \\
\hline \multicolumn{10}{|l|}{ Dietary patterns } \\
\hline Healthy conscious & $0.1 \dagger$ & $0.02,0.2$ & 0.1 & $-0 \cdot 1,0.2$ & 0.1 & $-0.02,0.2$ & $0.2^{\star \star}$ & $0.01,0.4$ & Reference \\
\hline Traditional & $0.1 \dagger$ & $0.03,0.2$ & $0 \cdot 2^{\star \star}$ & $0.02,0.3$ & -0.01 & $-0.1,0.2$ & $0.3^{\star \star \star}$ & $0.1,0.5$ & Reference \\
\hline Processed & -0.1 & $-0.2,-0.03$ & \multirow{2}{*}{\multicolumn{2}{|c|}{$\begin{array}{l}- \\
-\end{array}$}} & \multicolumn{2}{|c|}{-} & \multicolumn{2}{|c|}{-} & Reference \\
\hline Confectionery & -0.1 & $-0.1,0.03$ & & & \multicolumn{2}{|c|}{ - } & \multicolumn{2}{|c|}{-} & Reference \\
\hline Vegetarian & $0.3^{\star * \star}$ & $0.2,0.4$ & \multicolumn{2}{|r|}{$0.2,0.5$} & $0.3^{* * *}$ & $0.1,0.4$ & $0.5^{\star \star *}$ & $0.3,0.7$ & Reference \\
\hline
\end{tabular}

ED, eating disorders; AN, anorexia nervosa; BN, bulimia nervosa; $A N+B N$, both anorexia and bulimia nervosa.

Subgroup differences were not calculated if no statistically significant difference was present when comparing ED $v$. unexposed: ${ }^{* *} P \leq 0.01,{ }^{* \star *} P \leq 0.001$

† Subgroup differences were not calculated if no statistically significant difference was present when comparing ED $v$. unexposed $(P<0.1)$.

$\ddagger$ Adjusted for maternal age, maternal education and ethnicity.

$\S$ Comparing each group with the unexposed. 
Table 3. Use of fats, milk and caffeine: odds ratiosł ( $95 \%$ confidence intervals) from logistic regression for each group compared with unexposed women (Odds ratios and $95 \%$ confidence intervals)

\begin{tabular}{|c|c|c|c|c|c|c|c|c|c|}
\hline & \multicolumn{2}{|c|}{ ED $(n 414)$} & \multicolumn{2}{|c|}{ AN $(n 151)$} & \multicolumn{2}{|c|}{$\mathrm{BN}(n 186)$} & \multicolumn{2}{|c|}{$\mathrm{AN}+\mathrm{BN}(n 77)$} & \multirow[b]{2}{*}{$\begin{array}{l}\text { General population } \\
(n 9723)\end{array}$} \\
\hline & OR & $95 \% \mathrm{Cl}$ & OR & $95 \% \mathrm{Cl}$ & OR & $95 \% \mathrm{Cl}$ & OR & $95 \% \mathrm{Cl}$ & \\
\hline \multicolumn{10}{|l|}{ Fats (yes $v$. no) } \\
\hline Butter & $0.7^{\star \star \star}$ & $0.5,0.8$ & 0.8 & $0.5,1.1$ & $0.6^{\star \star}$ & $0.5,0.9$ & $0.6^{*}$ & $0.3,1 \cdot 0$ & Reference \\
\hline Margarine & $1.2 \dagger$ & $1 \cdot 0,1 \cdot 5$ & & & & & & & Reference \\
\hline Low-fat spread & $1 \cdot 1$ & $0.9,1.3$ & & & & & & & Reference \\
\hline Vegetable oil (sunflower or other) & $1 \cdot 1$ & $0.8,1.4$ & & & & & & & Reference \\
\hline \multicolumn{10}{|l|}{ Milk (usually/sometimes $v$. never) } \\
\hline Full-fat milk & $0.7^{\star \star \star}$ & $0.6,0.9$ & 0.9 & $0 \cdot 6,1 \cdot 2$ & $0 \cdot 6^{\star \star}$ & $0.5,0.8$ & 0.7 & $0.4,1.0$ & Reference \\
\hline Semi-skimmed milk & $1 \cdot 1$ & $0.9,1.4$ & & & & & & & Reference \\
\hline Skimmed milk & $1.4^{\star}$ & $1 \cdot 1,1 \cdot 8$ & $1.4^{\star}$ & $1 \cdot 0,2 \cdot 0$ & 1.3 & $1.0,1.9$ & $1.7^{\star}$ & $1 \cdot 0,2 \cdot 7$ & \\
\hline Soya milk & $3 \cdot 1^{\star \star \star}$ & $1 \cdot 8,5 \cdot 2$ & $5 \cdot 3^{\star \star \star}$ & $2 \cdot 8,10 \cdot 3$ & $1 \cdot 7$ & $0.6,4.6$ & 1.9 & $0.4,7.9$ & \\
\hline \multicolumn{10}{|l|}{ Takeaway meals/month } \\
\hline $1-4 /$ month & 1 & & & & & & & & Reference \\
\hline None/very few & $1 \cdot 2$ & $1.0,1.5$ & & & & & & & \\
\hline$>$ Once/week & $1 \cdot 3$ & $0.8,2 \cdot 2$ & & & & & & & \\
\hline \multicolumn{10}{|l|}{ Weekly caffeine dose } \\
\hline$<700 \mathrm{~g}$ & 1 & & 1 & & 1 & & 1 & & Reference \\
\hline $700-1400 \mathrm{~g}$ & 0.9 & $0 \cdot 7,1 \cdot 1$ & & & & & & & \\
\hline $1401-2099 \mathrm{~g}$ & 1.1 & $0.8,1.5$ & & & & & & & \\
\hline $2100-2449 \mathrm{~g}$ & 0.9 & $0.5,1.6$ & & & & & & & \\
\hline$\geq 2500 \mathrm{~g}$ & $1.9^{\star \star \star}$ & $1 \cdot 3,2 \cdot 8$ & $2 \cdot 6^{\star \star \star}$ & $1 \cdot 4,4 \cdot 8$ & 1.6 & $0.9,2.9$ & $2 \cdot 7^{*}$ & $1 \cdot 1,7 \cdot 0$ & \\
\hline
\end{tabular}

$\mathrm{ED}$, eating disorders; $\mathrm{AN}$, anorexia nervosa; $\mathrm{BN}$, bulimia nervosa; $\mathrm{AN}+\mathrm{BN}$, both anorexia nervosa and bulimia nervosa.

Subgroup differences were not calculated if no statistically significant difference was present when comparing ED $v$. unexposed: ${ }^{\star} P \leq 0.05$, ${ }^{* \star} P \leq 0.01,{ }^{\star \star \star} P \leq 0.001$.

† Subgroup differences were not calculated if no statistically significant difference was present when comparing ED $v$. unexposed $(P \leq 0 \cdot 1)$.

$\ddagger$ Adjusted for maternal age, maternal education and ethnicity.

there was evidence of a lower consumption of non-milk extrinsic sugars (for women with $\mathrm{BN}$ and $\mathrm{AN}+\mathrm{BN}$ ) and saturated fat (particularly women with lifetime $\mathrm{AN}$ and $\mathrm{AN}+\mathrm{BN}$ ) and more fibre (NSP) compared with unexposed women.

There was very little difference in terms of micronutrient intakes in pregnancy in women with lifetime ED compared with healthy women, consistent with Siega-Riz et al. ${ }^{(5)}$. In particular, no deficiencies in relation to vitamin and mineral intakes were shown for women with lifetime ED. This is encouraging, as it suggests that the majority of women with lifetime ED have adequate vitamin and mineral intake in pregnancy, and it might be secondary to general improved nutrition (as highlighted by the lack of difference in macronutrient intake).

The present study relied on a large general population sample of pregnant women, and it is the first study to investi-

Table 4. Nutrients: adjusted $B$ coefficients from ANOVA ( $B$ Coefficients and $95 \%$ confidence intervals)

\begin{tabular}{|c|c|c|c|}
\hline \multirow[b]{2}{*}{ Mean nutrient daily intakes } & \multicolumn{3}{|c|}{$\begin{array}{c}\text { ED } \\
(n \text { 404) }\end{array}$} \\
\hline & $B$ & $95 \% \mathrm{Cl}$ & $\begin{array}{l}\text { Unexposed } \\
\text { (n 9669) }\end{array}$ \\
\hline Energy (kJ) & $-186 \cdot 2^{*}$ & $-382 \cdot 8,10 \cdot 4$ & Reference \\
\hline Carbohydrates $\ddagger(\mathrm{g})$ & 1.0 & $-1 \cdot 2,3 \cdot 3$ & Reference \\
\hline Fatł (g) & -0.8 & $-1 \cdot 6,0 \cdot 1$ & Reference \\
\hline Protein $\ddagger(\mathrm{g})$ & 0.6 & $-0.5,1.7$ & Reference \\
\hline
\end{tabular}

$E D$, eating disorder.

${ }^{*} P<0.1$.

† Adjusted for maternal education, age and ethnicity.

$\ddagger$ Carbohydrate, fat and protein intakes were also adjusted for total energy. gate nutrition in the third trimester of pregnancy in women with lifetime ED. The sample of pregnant women was representative of the general population in the geographical area under study.

The main weakness in the present study relates to the ascertainment of ED history. This was obtained by selfreport. In particular, one question ascertained the lifetime history of ED, resulting in a possible misclassification of exposure. It is likely that this represents an underestimate of ED, given the tendency of screening measures for ED in community samples to miss cases. However, self-reported ED was validated using behavioural and cognitive characteristics in this sample ${ }^{(1)}$. Moreover, self-report of ED has been shown to be comparable to longer and widely used ED screening instruments for screening purposes in general population studies ${ }^{(21)}$. In addition, the prevalence of selfreported $\mathrm{ED}(3.7 \%)$ in this sample is consistent with general population lifetime prevalence.

Unfortunately, it was not possible to ascertain active $v$. lifetime ED during pregnancy.

The other major weakness was that standard portion sizes were used to assess nutrient intakes from the FFQ. Clinically, women with past/active ED often report difficulties in determining what was a good portion size. It is possible that women with lifetime AN ate small portions and those with BN binged on certain foods. The FFQ used for the present study only asked about the frequency with which each food group was consumed. It is possible that women with ED, albeit eating a good-quality diet, did not eat enough in quantity. 


\section{Conclusions}

Despite being more likely to be vegetarian and consuming less meat, women with lifetime ED had similar protein, fat and carbohydrate intake compared with unexposed women. In general, good macronutrient and vitamin and mineral intake were apparent. This is encouraging and suggests that the quality of maternal diets in pregnancy was reasonable in women with lifetime ED. There was some evidence that they made more healthy choices compared with unexposed women. However, we were unable to account for portion sizes. Future analyses should investigate actual gestational weight gain in this sample. A high caffeine intake was shown in women with ED and future research needs to clarify the impact of this on fetal growth.

\section{Acknowledgements}

We are extremely grateful to all the families who took part in this study, the midwives for their help in recruiting them, and the whole ALSPAC team, which includes interviewers, computer and laboratory technicians, clerical workers, research scientists, volunteers, managers, receptionists and nurses. The UK Medical Research Council, the Wellcome Trust and the University of Bristol provided core support for the ALSPAC. This study was funded by the National Alliance for Schizophrenia and Depression, the Department of Health via the ARIADNE programme (Applied Research into Anorexia Nervosa and Not Otherwise Specified Eating Disorders) and a Department of Health National Institute for Health Research (NIHR) Programme Grant for Applied Research (reference no. RP-PG-0606-1043) to U. Schmidt, J. Treasure, K. Tchanturia, H. Startup, S. Ringwood, S. Landau, M. Grover, I. Eisler, I. Campbell, J. Beecham, M. Allen, G. Wolff. The views expressed herein are not necessarily those of the Department of Health/NIHR. N. M. devised the study hypothesis, analysed the data and wrote the paper, and has primary responsibility for the final content. K. N. and P. E. were involved in the original cohort design, performed the data extraction and the general data analyses, helped with the data interpretation and revised the manuscript. U. N. advised on the data analytic strategies and advised on the interpretation of the data analyses. J. L. T. supervised the data analyses, the interpretation of the data and revised the manuscript. The authors declare that there are no conflicts of interest.

Supplementary tables are available online at http://www. journals.cambridge.org/bjn

\section{References}

1. Micali N, Simonoff E \& Treasure J (2007) Risk of major adverse perinatal outcomes in women with eating disorders. Br J Psychiatry 190, 255-259.

2. Bulik CM, Von Holle A, Siega-Riz AM, et al. (2009) Birth outcomes in women with eating disorders in the Norwegian mother and child cohort study (MoBa). Int J Eat Disord 42 , 9-18.

3. Ekeus C, Lindberg L, Lindblad F, et al. (2006) Birth outcomes and pregnancy complications in women with a history of anorexia nervosa. Br J Obstet Gynaecol 113, 925-929.

4. Sollid CP, Wisborg K, Hjort J, et al. (2004) Eating disorder that was diagnosed before pregnancy and pregnancy outcome. Am J Obstet Gynecol 190, 206-210.

5. Siega-Riz AM, Haugen M, Meltzer HM, et al. (2008) Nutrient and food group intakes of women with and without bulimia nervosa and binge eating disorder during pregnancy. $A m \mathrm{~J}$ Clin Nutr 87, 1346-1355.

6. Micali N \& Treasure J (2009) Biological effects of a maternal ED on pregnancy and foetal development: a review. Eur Eat Disord Rev 17, 448-454.

7. Golding J, Pembrey M \& Jones R (2001) ALSPAC, the Avon Longitudinal Study of Parents and Children, I: study methodology. Paediatr Perinat Epidemiol 15, 74-87.

8. Northstone K, Emmett PM \& Rogers I (2008) Dietary patterns in pregnancy and associations with nutrient intakes. $\mathrm{Br} J$ Nutr 99, 406-415.

9. Rogers IS \& Emmett PM (1998) Diet during pregnancy in a population of pregnant women in South West England. ALSPAC Study Team. Avon Longitudinal Study of Pregnancy and Childhood. Eur J Clin Nutr 52, 246-250.

10. Gregory J, Foster K, Tyler H, et al. (1990) Classification and Types of Diet. The Dietary and Nutritional Survey of British Adults, chapter 13. London: HMSO.

11. StataCorp (2007) Stata 10 for Windows [Computer Program]. College Station, TX: StataCorp.

12. Holm S (1979) A simple sequentially rejective multiple test procedure. Scand J Stat 6, 65-70.

13. Bender R \& Lange S (2001) Adjusting for multiple testing when and how? J Clin Epidemiol 54, 343-349.

14. Craig WJ \& Mangels AR (2009) Position of the American Dietetic Association: vegetarian diets. J Am Diet Assoc 109, 1266-1282.

15. Guerrero-Bosagna CM, Sabat P, Valdovinos FS, et al. (2008) Epigenetic and phenotypic changes result from a continuous pre and post natal dietary exposure to phytoestrogens in an experimental population of mice. BMC Physiol 15, 8-17.

16. FSA (Food Standards Agency) (2008) Food Standards Agency Publishes New Caffeine Advice for Pregnant Women. http:// www.food.gov.uk/news/pressreleases/2008/nov/caffeineadvice (accessed 24 February 2009).

17. Hart S, Abraham S, Luscombe G, et al. (2005) Fluid intake in patients with eating disorders. Int J Eat Disord 38, 55-59.

18. Striegel-Moore RH, Franko DL, Thompson D, et al. (2006) Caffeine intake in eating disorders. Int $J$ Eat Disord 39, $162-165$.

19. Parazzini F, Chiaffarino F \& Chatenoud L (2005) Maternal coffee drinking in pregnancy and risk of small for gestational age birth. Euro J Clin Nutr 59, 299-301.

20. Bakker R, Steegers EA, Obradov A, et al. (2010) Maternal caffeine intake from coffee and tea, fetal growth, and the risks of adverse birth outcomes: the Generation R Study. Am J Clin Nutr 91, 1691-1698.

21. Keski-Rahkonen A, Sihvola E, Raevuori A, et al. (2006) Reliability of self-reported eating disorders: optimizing population screening. Int J Eat Disord 39, 754-762. 